\title{
Human cleaving embryos enable robust homozygotic nucleotide substitutions by base editors
}

\author{
Meiling Zhang ${ }^{1,2+}$, Changyang Zhou ${ }^{3,8+}$, Yu Wei ${ }^{3+}$, Chunlong $X u^{3+}$, Hong Pan ${ }^{3}$, Wenqin Ying ${ }^{3}$, Yidi Sun ${ }^{9}$, Yun Sun ${ }^{1,2}$, \\ Qingquan Xiao ${ }^{3,8}$, Ning Yao ${ }^{1,2}$, Wanxia Zhong ${ }^{1,2}$, Yun $\mathrm{Li}^{1,2}$, Keliang Wu ${ }^{5,6,7}$, Gao Yuan ${ }^{5,6,7}$, Shoukhrat Mitalipov ${ }^{4,5^{*}}$, \\ Zi-jiang Chen ${ }^{1,2,5,6,7^{*}}$ and Hui Yang ${ }^{3^{*}}$
}

\begin{abstract}
Base editing installs a precise nucleotide change in specific gene loci without causing a double-strand break. Its efficiency in human embryos is generally low, limiting its utility in functional genetic studies. Here, we report that injecting base editors into human cleaving two-cell and four-cell embryos results in much higher (up to 13-fold) homozygotic nucleotide substitution efficiency as opposed to Mll oocytes or zygotes. Furthermore, as a proof-ofprinciple study, a point mutation can be efficiently corrected by our method. Our study indicates that human cleaving embryos provide an efficient base editing window for robust gene disruption and correction.
\end{abstract}

Keywords: Base editing, Human cleaving embryos, Homozygotic nucleotide substitution

\section{Background}

Base editors, enabling single nucleotide conversion without causing double-strand breaks, have been successfully applied for base correction in mouse and human embryos [1-5]. In contrast to the mouse, base-editing efficiency in human embryos is generally low (below 30\%) that frequently leads to mosaicism and limits the utility of current base editing methods for gene functional study in human embryos (Additional file 1). Several species-specific differences in early embryonic development may account for low efficiency of BEs in human embryos. Here, we investigate whether injecting base editors into human embryos at different stages has an influence on base-editing efficiency.

\footnotetext{
*Correspondence: mitalipo@ohsu.edu; chenzijiang@hotmail.com; huiyang@ion.ac.cn

${ }^{+}$Meiling Zhang, Changyang Zhou, Yu Wei, and Chunlong Xu contributed equally to this work

${ }^{4}$ Center for Embryonic Cell and Gene Therapy, Oregon Health \& Science University, 3303 Southwest, Bond Avenue, Portland, OR 97239, USA

${ }^{1}$ Center for Reproductive Medicine, Ren Ji Hospital, School of Medicine, Shanghai Jiao Tong University, Shanghai, China

${ }^{3}$ Institute of Neuroscience, State Key Laboratory of Neuroscience, Key Laboratory of Primate Neurobiology, CAS Center for Excellence in Brain Science and Intelligence Technology, Shanghai Institutes for Biological Sciences, Chinese Academy of Sciences, Shanghai 200031, China Full list of author information is available at the end of the article
}

\section{Results and discussion}

To test the base-editing system in human embryos, we initially injected BE3 mRNA and sgRNA into one-cell embryo (zygote stage) (Fig. 1a, b and Additional file 1: Supplementary Methods) to induce G>A conversions (g.97G $>\mathrm{A}, \mathrm{G}_{8} ;$ g.98G $>\mathrm{A}, \mathrm{G}_{7}$ ) in exon 1 of $\beta$-globin $(H B B)$ gene (Fig. 1a-c). BE3 mRNA and sgRNAs were co-injected into the cytoplasm of 3PN zygotes approximately $24 \mathrm{~h}$ post fertilization (one-cell stage) (Fig. 1b). Injected zygotes were cultured to the eight-cell stage and used for on-target deep sequencing analysis (Additional file 5: Table S3. Primers used in the study). Although expected Trp16 to Stop conversions (g.97G > A or g.98G > A) in the $H B B$ locus were observed in some blastomeres of all injected embryos $(n=6)$, base-editing frequency was relatively low with high mosaicism by one-cell injection (27.8 $\pm 9.7 \%$; Fig. 1c, Additional file 2: Figure S1a, and Additional file 3: Table S1).

The onset of zygotic gene activation (ZGA) in human embryos (four- to eight-cell stage) is typically later than that of mouse embryos (two-cell stage) [6, 7]. Therefore, we decided to test the conversion efficiency in cleaving human embryos by injecting BE3 mRNA and corresponding sgRNA into each blastomere of the two-cell or four-cell stage embryos (Fig. 1a) and measuring outcomes

(c) The Author(s). 2019 Open Access This article is distributed under the terms of the Creative Commons Attribution 4.0 International License (http://creativecommons.org/licenses/by/4.0/), which permits unrestricted use, distribution, and reproduction in any medium, provided you give appropriate credit to the original author(s) and the source, provide a link to the Creative Commons license, and indicate if changes were made. The Creative Commons Public Domain Dedication waiver (http://creativecommons.org/publicdomain/zero/1.0/) applies to the data made available in this article, unless otherwise stated. 


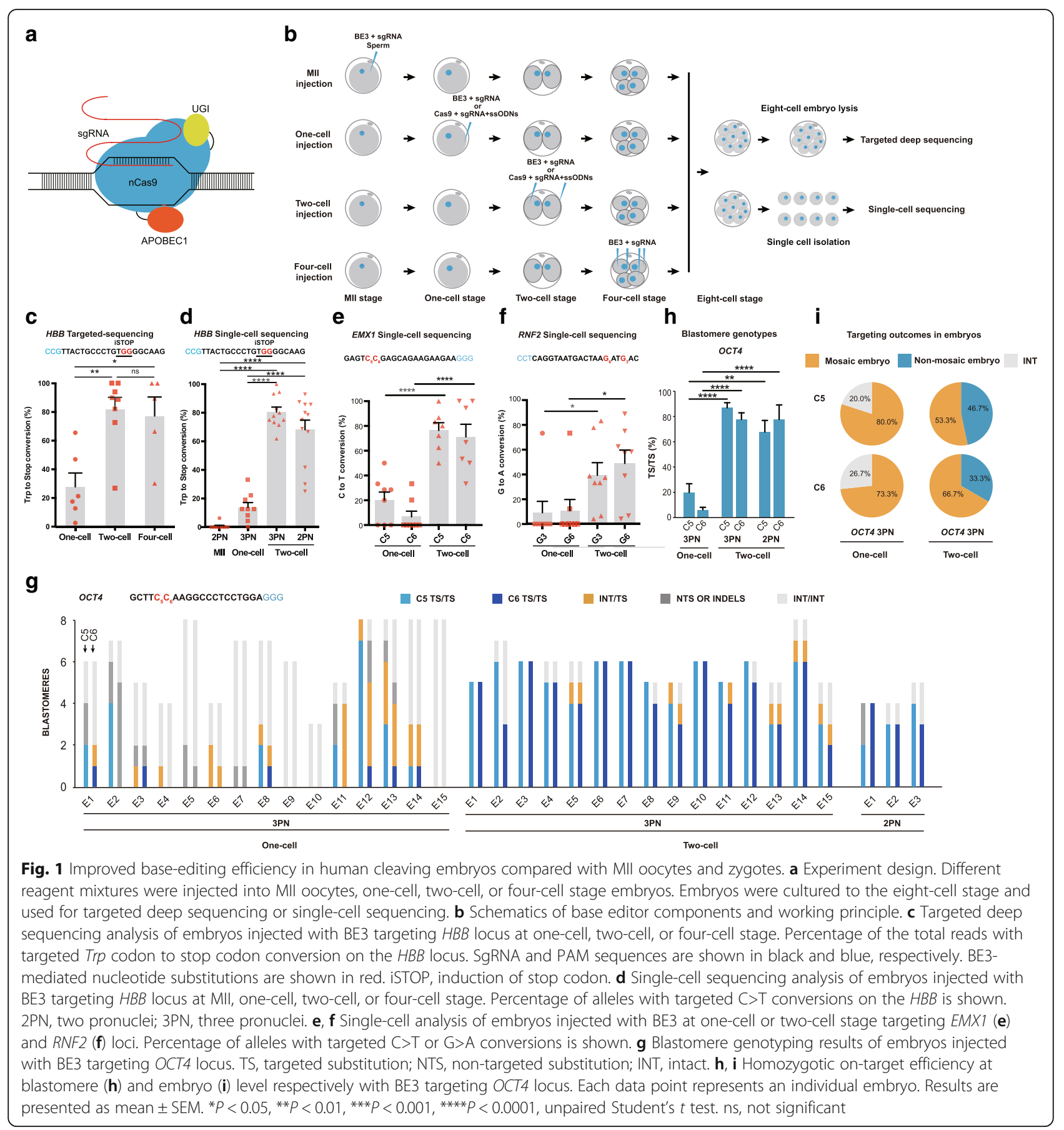

in eight-cell embryos. Remarkably, the efficiency of targeted $\mathrm{G}>\mathrm{A}$ conversions was greatly increased and reached $82.6 \pm 8.7 \%$ when injected into two-cell and $77.2 \pm 13.3 \%$ into four-cell embryos in contrast with low efficiency in one-cell embryo (Fig. 1c, Additional file 2: Figure S1a, and Additional file 3: Table S1). Furthermore, we corroborated this finding by targeted g.22909C $>\mathrm{T}\left(\mathrm{C}_{5}\right)$ and g.22910C $>\mathrm{T}$ $\left(\mathrm{C}_{6}\right)$ conversions in the exon 3 of EMX1 gene and confirmed a significant increase in base-editing efficiency when treated cleaving human embryos as opposed to zygotes (Additional file 2: Figure S1b,c and Additional file 3: Table S1). We also used single-blastomere sequencing to analyze each cell of the multicellular embryo, which allows us to define the allelic targeting profile of each blastomere. After BE3 injection, each blastomere from the eight-cell embryos was isolated and individually sequenced (Fig. 1b). The efficiency of base editing at allelic level obtained from single-blastomere sequencing was consistent with that of deep sequencing analysis in $H B B$, confirming higher efficiency in two-cell injection than that in one-cell injection 
$(80.50 \pm 3.43 \%$ in two-cell versus $13.84 \pm 3.33 \%$ in one-cell injection) (Fig. 1d and Additional file 4: Table S2). We also tested the base-editing efficiency in diploid embryos (2PN) at $H B B$ locus and found similar results with 3PN ones (Fig. 1d; Additional file 4: Table S2; Additional file 5). It has been reported most zygotes had completed $\mathrm{S}$ phase of the cell cycle and DNA replication and likely produced four alleles for targeting, leading to high mosaicism $[8,9]$. Therefore, we co-injected BE3 into MII oocytes with sperm during fertilization by intracytoplasmic sperm injection (ICSI) (Fig. 1b). Unexpectedly, MII oocyte injection resulted in much a lower base-editing frequency $(0.63 \%)$ compared to pronuclear stage zygotes (13.8\%) (Fig. 1d and Additional file 4: Table S2).

Though two-cell injection improved on-target efficiency compared with one-cell injection, indels or non-target substitution frequency stayed similarly low with less than 5\% in both stages (Additional file 2: Figure S1e and Additional file 4: Table S2). Moreover, development competency of treated embryos to the eight-cell stage for $H B B$ locus was not affected and comparable to uninjected control group (Additional file 2: Figure S1f). With experiments on more loci, we could also achieve the improved base-editing efficiency by two-cell injection at EMX1, RNF2, and OCT4 loci (Fig. 1b-d, Additional file 2: Figure S1d, and Additional file 4: Table S2). Besides BE3-mediated $\mathrm{C}>\mathrm{T}$ and $\mathrm{G}>\mathrm{A}$ conversion, we additionally examined the efficiency of $A>G$ and $T>C$ conversions in cleaving human embryos by $\mathrm{ABE}$ system targeting three separate genomic loci, site 2 , site 4 , and site 6 [10]. Like the results in BE3 experiments, injection of ABE mRNA and corresponding sgRNA into two-cell human embryos resulted in significantly higher $A>G$ conversions than in zygotes (Additional file 2: Figure S2a-c, Figure S3a-c and Additional file 4: Table S2).

After higher base-editing efficiency in cleaving embryos was verified at the allelic level, we further analyzed the homozygotic targeting efficiency in each blastomere. In this regard, we chose OCT4 locus at the exon 1 to investigate the simultaneous induction of g.187C $>\mathrm{T} \quad\left(\mathrm{C}_{5}\right)$ and g.188C $>\mathrm{T}\left(\mathrm{C}_{6}\right)$ conversions at three parental alleles by injecting BE3 into human two-cell embryos derived from 3PN zygotes (Fig. 1g). Single-blastomere analysis revealed that $87.4 \%$ (76 out of 87 blastomeres) of blastomeres carried desired $\mathrm{C}>\mathrm{T}$ substitutions at the g.187C locus and $78.2 \%$ ( 68 out of 87 blastomeres) at the g.188C position in all three alleles (tri-allelic base substitutions) (Fig. $1 \mathrm{~g}$, $\mathrm{h}$ and Additional file 4: Table S2). By contrast, only $22.3 \%$ and $6.4 \%$ of blastomeres derived from conventional zygote injection carried g.187C $>\mathrm{T}$ and g.188C $>\mathrm{T}$ tri-allelic base substitutions, respectively (Fig. 1g, h and Additional file 4: Table S2). Besides 3PN embryos, we also targeted the same OCT4 locus in two-cell embryos derived from normally fertilized (2PN) zygotes. The percentage of homozygotic targeted blastomere was $68.3 \%$ for the g.187C locus and $78.3 \%$ for the g.188C site, comparable to those derived from abnormally fertilized 3PN embryos (Fig. 1g, h and Additional file 4: Table S2). Remarkably, 5 out of the total 15 two-cell injected embryos derived from 3PN zygotes carried homozygous $\mathrm{C}>\mathrm{T}$ substitutions at both g.187C and g.188C loci, whereas none of one-cell injected embryos have such complete editing in each blastomere (Fig. 1i).

In addition to OCT4 locus, we also found significant improvement of homozygotic conversion efficiency in other loci including $H B B, E M X 1$ by two-cell injection compared to one-cell injection (Additional file 2: Figure S4a-d and Additional file 4: Table S2). These results indicate that base editors injection in cleaving embryos could efficiently induce nucleotide substitutions simultaneously in all parental alleles in a single blastomere, suggesting potential applications to interrogate the causality between homozygous point mutations and corresponding phenotype in human embryos.

We next tested whether base editors could be used for correcting point mutation and interrogating causality between mutations and corresponding phenotypes in early human embryonic development. As a proof-of-concept study, we chose a previously identified c.299A $>\mathrm{G}$ mutation in $M U T$ gene encoding methylmalonyl CoA mutase for the correction experiment. Homozygous c.299A $>G$ substitution in $M U T$ leads to methylmalonic acidemia, a condition characterized by feeding difficulties, developmental delay, and long-term health problems [11]. We identified an adult male with the heterozygous c. $299 \mathrm{~A}>\mathrm{G}(\mathrm{g} .4133 \mathrm{~A}>\mathrm{G})$ mutation, and he consented to donate a semen sample. In contrast to mutant c.299A $>G$ allele, normal wild-type gene carried two linked neutral SNPs (NC_000006.12, g.2259C $>\mathrm{T}$; NC_000006.12, g.2654C >G) (Fig. 2a and Additional file 2: Figure S5a, b).

We then fertilized in vitro matured MII oocytes with the carrier sperms and injected BE3 mRNA with sgRNA into two-cell embryos (Fig. 2b). Embryos were further cultured into the eight-cell stage and used for single-blastomere analysis. Original heterozygous mutant embryos $\left(\mathrm{MUT}^{+/ \mathrm{C} .299 \mathrm{~A}>\mathrm{G}}\right)$ produced from the mutant sperm were identified and separated from wild-type $\left(\mathrm{MUT}^{+/+}\right)$embryos by the presence of the linked SNPs. In intact controls, $50 \%(8 / 16)$ of embryos were $\mathrm{MUT}^{+/+}$ while the other half $(8 / 16)$ were $\mathrm{MUT}^{+/ \mathrm{C} \cdot 299 \mathrm{~A}>\mathrm{G}}$ (Additional file 2: Figure S5c and Additional file 4: Table S2). In embryos injected with BE3 mRNA and sgRNA, 10 out of total 15 (66.7\%) were uniformly homozygous $\left(\mathrm{MUT}^{+/+}\right)$, among which 2 embryos were fertilized by mutant sperm (MUT ${ }^{\text {c.299A }>\text { G }}$ ) (Fig. 2c). The remaining 5 embryos (33.3\%) were mosaic carrying 2 types of blastomeres, $\mathrm{MUT}^{+/+}$and $\mathrm{MUT}^{+/ \mathrm{C} .299 \mathrm{~A}>\mathrm{G}}$ (Fig. 2c). However, in the 5 mosaic and 2 completely corrected mutant embryos, $87.6 \%$ of analyzed alleles were WT and $75.7 \%$ (28/ 


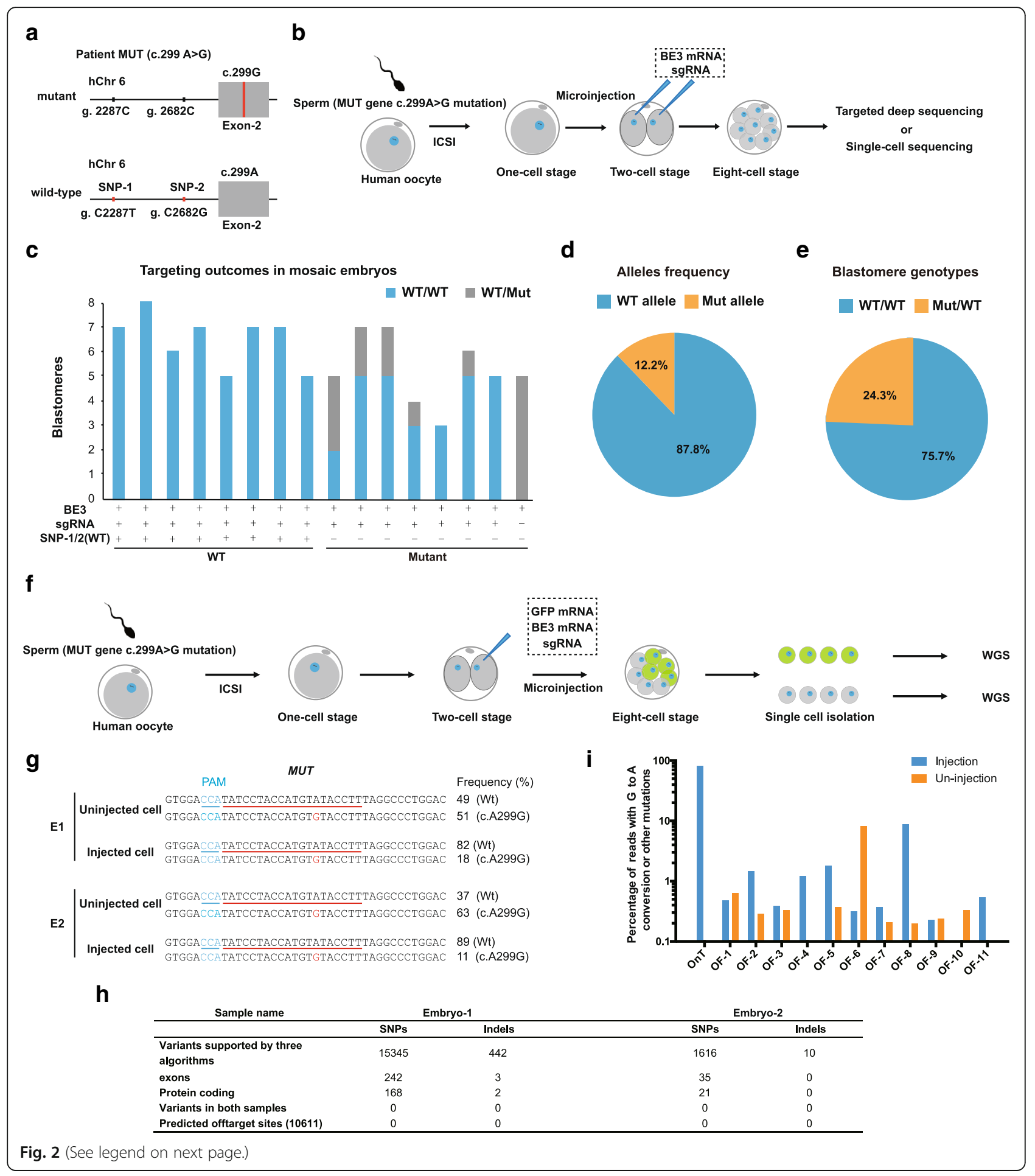




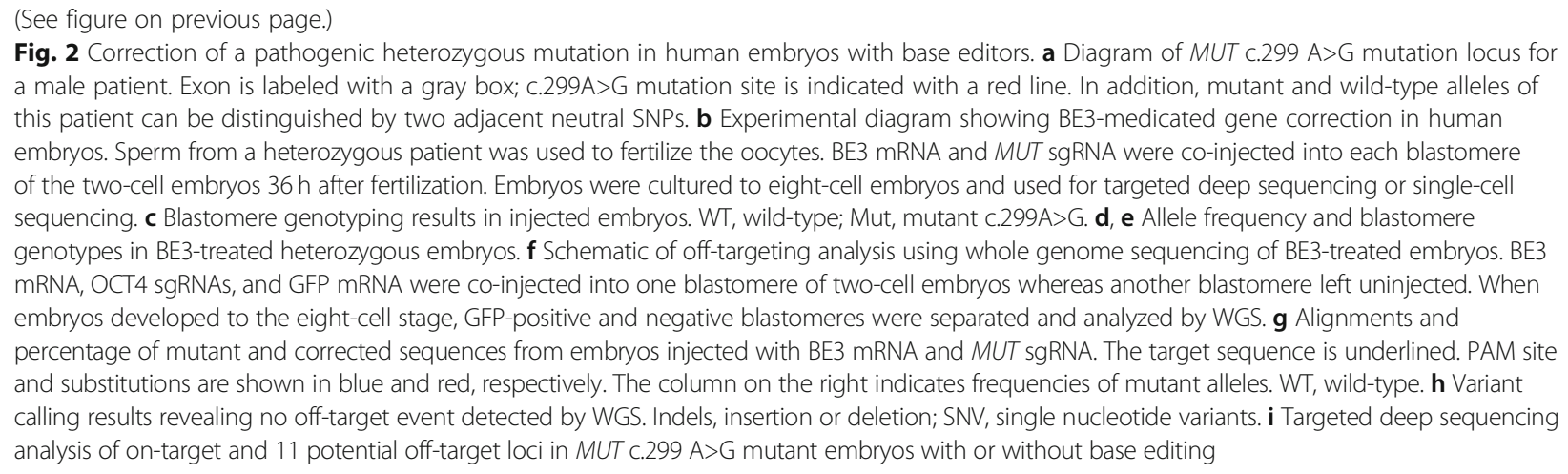

35) of blastomeres became homozygous with only wild-type genotype of $\mathrm{MUT}^{+/+}$, indicating the proper correction of the mutant paternal allele with base editing (Fig. 2d, e). Furthermore, all BE3-treated embryos derived from the WT sperm were uniformly homozygous $\left(\mathrm{MUT}^{+/+}\right)$without any misconversions or indels indicating high specificity of base editing (Fig. 2c).

We next investigated if base editing induced any off-target alterations. To eliminate the differences in the genetic background between the gene-edited and control embryos, BE3, GFP mRNA, and sgRNA were co-injected into only one blastomere of two-cell embryos while leaving another one uninjected (Fig. 2f). Injected blastomeres were identified by GFP expression $\left(\mathrm{GFP}^{+}\right)$in eight-cell embryos. Whole genome sequencing (WGS) was performed on both $\mathrm{GFP}^{+}$and $\mathrm{GFP}^{-}$cells, and multiple variant-calling software pipelines were used to ensure reliable identification of indels and single nucleotide variants (SNVs) (Fig. $2 \mathrm{~g}$, h). In the results from two BE3-edited embryos analyzed, we found neither variants shared in two BE3-edited embryos nor variants in 10,611 predicted off-target sites (Fig. 2h). We also performed targeted deep sequencing to verify the top 11 predicted off-target sites and still found no evidence for off-target mutations (Fig. 2i and Additional file 4: Table S2).

In summary, we showed that the delivery of base editors into cleaving two-cell or four-cell human embryos resulted in much higher homozygotic nucleotide conversion rates, possibly due to more compact chromatin in human zygotes and massive RNA degradation event around zygote cleavage stage (Additional file 2: Figure S6a, b) [12]. A recent work reported the correction of a Marfan syndrome (MFS) pathogenic mutation in embryos by base editing [5]. However, the conclusions were untenable due to the low number of embryos and inadequate experimental design and data analysis (Additional file 2: Figure S7a, b). Notably, two recent studies have reported that BE3 generates substantial off-target mutations in mouse embryos and rice [13, 14]. However, no overlapped mutation was found in our study between any two individual embryos and very few of them located on exon, unlikely affecting the base editor application for gene function study during human embryonic development. Certainly, it will be highly desirable to explore and use base editors of high efficiency and fidelity for gene manipulation in human embryos in the future.

\section{Methods}

Retrieval of 3PN embryos during in vitro fertilization

The COCs were inseminated in 4-well plates with approximately 100,000 motile spermatozoa for each oocyte. Approximately $18-20 \mathrm{~h}$ after fertilization, we collect 3PN embryos for the experiment.

\section{Derivation of 2PN embryos by ICSI}

Immature MI oocytes were collected from patients for IVF or ICSI treatment. MI oocytes were cultured in IVM medium in vitro for the first polar body extrusion by observation every $2 \mathrm{~h}$. ICSI was performed $3 \mathrm{~h}$ after polar body extrusion.

\section{Injection of base editors into embryos}

For one-cell injection, the mixture of BE3/ABE mRNA $(100 \mathrm{ng} / \mu \mathrm{l})$ and sgRNA $(50 \mathrm{ng} / \mu \mathrm{l})$ was injected into the cytoplasm of the zygotes $24 \mathrm{~h}$ after fertilization. For two-cell or four-cell injection, the mixture of $\mathrm{BE} 3 / \mathrm{ABE}$ mRNA $(100 \mathrm{ng} / \mu \mathrm{l})$ and sgRNA $(50 \mathrm{ng} / \mu \mathrm{l})$ was injected into every blastomere of two-cell or four-cell embryos 36 or $44 \mathrm{~h}$ after fertilization, respectively.

\section{Single-blastomere sequencing analysis}

Individual blastomeres were put into PCR tubes with $1.5 \mu \mathrm{l}$ embryo lysis buffer and used for nest PCR. The PCR product was analyzed by Sanger sequencing to detect the efficiency of base editing.

\section{Statistical analysis}

All statistical values were presented as mean \pm SEM. Differences between datasets were considered to be significant at $P$ value less than 0.05 . All the statistic tests were conducted with Student $t$ test unless otherwise stated. 


\section{Additional files}

Additional file 1: Supplementary methods. (DOCX $26 \mathrm{~kb}$ )

Additional file 2: Figure S1. Highly increased base-editing efficiency in cleaving human embryos compared with zygote. Figure S2. Improved base-editing efficiency in human cleaving embryos with ABEs. Figure S3. Frequency of indel mutations and off-targeted nucleotide substitutions in human embryos injected by base editors. Figure S4. Targeting homozygous loci in human embryos with base editors. Figure S5. Identification of SNPs in the patient distinguishing MUT c.299A>G from WT allele. Figure S6. Cleaving embryos have a higher level of GFP fluorescence than one-cell embryo $24 \mathrm{~h}$ post-mRNA injection. Figure S7. Comparison results of base editing in human embryos in two studies. (PDF $1771 \mathrm{~kb}$ )

Additional file 3: Table S1. Targeted deep sequencing results. (XLSX $17 \mathrm{~kb}$ )

Additional file 4: Table S2. Single-cell sequencing results. (XLSX 54 kb)

Additional file 5: Table S3. Primers used in the study. (XLSX $12 \mathrm{~kb}$ )

Additional file 6: Review history. (DOCX $33 \mathrm{~kb}$ )

\section{Abbreviations}

2PN/3PN: Two/three pronuclei; ABE: Adenine base editor; BEs: Base editors; DSB: Double-strand break; ICSI: Intracytoplasmic sperm injection; IVF: In vitro fertilization; MFS: Marfan syndrome; MII: Metaphase II; SNP: Single nucleotide polymorphism; SNVs: Single nucleotide variants; UGI: UNG inhibitory protein; WGS: Whole genome sequencing; ZGA: Zygotic gene activation

\section{Acknowledgements}

We greatly thank the technician staff at Optical Imaging Facility in the Institute of Neuroscience. We appreciate Miss Linxiao Han and Miss Yu Wei for their help with the genotyping experiments.

\section{Review history}

The review history is available in Additional file 6.

\section{Funding}

This work was supported by the R\&D Program of China (2018YFC2000100 and 2017YFC1001302 to HY; 2016YFC1000604 to ZJC), CAS Strategic Priority Research Program (XDB32060000), National Natural Science Foundation of China (31871502, 31522037), Shanghai Municipal Science and Technology Major Project (2018SHZDZX05), Shanghai City Committee of Science and Technology Project (18411953700, 18JC1410100), and Shanghai Key Laboratory for Assisted Reproduction and Reproductive Genetic (17DZ2271100).

\section{Availability of data and materials}

The datasets generated during and/or analyzed during the current study are available in the SRA repository under accession number SRP136376 [15].

\section{Authors' contributions}

$C-Y Z$ and $M-L Z$ designed and performed the experiments. $Y W$ performed the human embryo injection. HP, W-QY, and Q-QX performed the genotyping. YDS performed the data analysis. YS, NY, WZ, YL, KW, and GY performed the human embryos collection. HY, Z-JC, and SM supervised the project. HY, SM, and C-LX wrote the paper. All authors read and approved the final manuscript.

\section{Ethics approval and consent to participate}

This study was approved by the ART Ethics Committee of Ren Ji Hospital, Shanghai Jiao Tong University (approval reference number: 2017112406 for tri-pronuclear zygotes and immature oocytes). The review of research project application was conducted by independent experts in the ART Ethics Committee. Our study has strictly followed the guidelines on human stem cells and embryo research in China (in accordance with 2016 ISSCR guidelines for stem cell Research and Clinical Applications of Stem (ells) and been ethically inspected. This study used abnormally fertilized tri-pronuclear (3PN) human zygotes, and immature oocytes, routinely discarded during IVF treatments, were donated for this study by consented IVF patients at Ren Ji
Hospital. Normal sperm and sperm with mutation in MUT gene were donated by volunteer donors after obtaining formal consent. All donations of sperm or embryos were voluntary without any financial inducement. Scientific publications for this study were formally allowed by all donors under consent statements. The consent forms related to this study were provided for the inspection without revealing donor identities during journal peer-review process by following confidential regulation rules in the Ren Ji Hospital. All experimental methods in the study abided by the Helsinki Declaration.

\section{Competing interests}

The authors declare that they have no competing interests.

\section{Publisher's Note}

Springer Nature remains neutral with regard to jurisdictional claims in published maps and institutional affiliations.

\section{Author details}

${ }^{1}$ Center for Reproductive Medicine, Ren Ji Hospital, School of Medicine, Shanghai Jiao Tong University, Shanghai, China. ${ }^{2}$ Shanghai Key Laboratory for Assisted Reproduction and Reproductive Genetics, Shanghai 200127, China. ${ }^{3}$ Institute of Neuroscience, State Key Laboratory of Neuroscience, Key Laboratory of Primate Neurobiology, CAS Center for Excellence in Brain Science and Intelligence Technology, Shanghai Institutes for Biological Sciences, Chinese Academy of Sciences, Shanghai 200031, China. ${ }^{4}$ Center for Embryonic Cell and Gene Therapy, Oregon Health \& Science University, 3303 Southwest, Bond Avenue, Portland, OR 97239, USA. ${ }^{5}$ Center for Reproductive Medicine, Shandong Provincial Hospital Affiliated to Shandong University, Jinan, Shandong, China. ${ }^{6}$ National Research Center for Assisted Reproductive Technology and Reproductive Genetics, Jinan, China. ${ }^{7}$ The Key laboratory for Reproductive Endocrinology of Ministry of Education, Jinan 250021,

Shandong, China. ${ }^{8}$ College of Life Sciences, University of Chinese Academy of Sciences, Beijing 100049, China. ${ }^{9}$ Key Lab of Computational Biology, CAS-MPG Partner Institute for Computational Biology, Shanghai Institutes for Biological Sciences, Chinese Academy of Sciences, Shanghai 200031, China.

Received: 18 January 2019 Accepted: 25 April 2019

Published online: 22 May 2019

\section{References}

1. Zhou C, Zhang M, Wei Y, Sun Y, Sun Y, Pan H, Yao N, Zhong W, Li Y, Li W, et al. Highly efficient base editing in human tripronuclear zygotes. Protein Cell. 2017;8(10):772-5.

2. Li G, Liu Y, Zeng Y, Li J, Wang L, Yang G, Chen D, Shang X, Chen J, Huang $X$, Liu J. Highly efficient and precise base editing in discarded human tripronuclear embryos. Protein Cell. 2017:8(10):776-9.

3. Liang $P$, Ding $C$, Sun $H$, Xie X, Xu Y, Zhang X, Sun Y, Xiong Y, Ma W, Liu Y, et al. Correction of beta-thalassemia mutant by base editor in human embryos. Protein Cell. 2017:8:811-22.

4. Kim K, Ryu SM, Kim ST, Baek G, Kim D, Lim K, Chung E, Kim S, Kim JS. Highly efficient RNA-guided base editing in mouse embryos. Nat Biotechnol. 2017; 35:435-7.

5. Zeng Y, Li J, Li G, Huang S, Yu W, Zhang Y, Chen D, Chen J, Liu J, Huang X. Correction of the Marfan syndrome pathogenic FBN1 mutation by base editing in human cells and heterozygous embryos. Mol Ther. 2018;26(11): 2631-7.

6. Braude $\mathrm{P}$, Bolton $\mathrm{V}$, Moore $\mathrm{S}$. Human gene expression first occurs between the four- and eight-cell stages of preimplantation development. Nature. 1988;332:459-61.

7. Flach G, Johnson MH, Braude PR, Taylor RA, Bolton VN. The transition from maternal to embryonic control in the 2-cell mouse embryo. EMBO J. 1982;1: 681-6.

8. Zuo E, Cai YJ, Li K, Wei Y, Wang BA, Sun Y, Liu Z, Liu J, Hu X, Wei W, et al. One-step generation of complete gene knockout mice and monkeys by CRISPR/Cas9-mediated gene editing with multiple sgRNAs. Cell Res. 2017; 27(7):933-45.

9. Ma H, Marti-Gutierrez N, Park SW, Wu J, Lee Y, Suzuki K, Koski A, Ji D, Hayama T, Ahmed R, et al. Correction of a pathogenic gene mutation in human embryos. Nature. 2017;548:413-9. 
10. Gaudelli NM, Komor AC, Rees HA, Packer MS, Badran AH, Bryson DI, Liu DR. Programmable base editing of A.T to G.C in genomic DNA without DNA cleavage. Nature. 2017;551:464-71.

11. Acquaviva C, Benoist JF, Pereira S, Callebaut I, Koskas T, Porquet D, Elion J. Molecular basis of methylmalonyl-CoA mutase apoenzyme defect in 40 European patients affected by mut(o) and mut- forms of methylmalonic acidemia: identification of 29 novel mutations in the MUT gene. Hum Mutat. 2005;25:167-76.

12. Lee MT, Bonneau AR, Giraldez AJ. Zygotic genome activation during the maternal-to-zygotic transition. Annu Rev Cell Dev Biol. 2014;30:581-613.

13. Zuo E, Sun Y, Wei W, Yuan T, Ying W, Sun H, Yuan L, Steinmetz LM, Li Y, Yang $\mathrm{H}$. Cytosine base editor generates substantial off-target singlenucleotide variants in mouse embryos. Science. 2019;364(6437):289-92.

14. Jin S, Zong Y, Gao Q, Zhu Z, Wang Y, Qin P, Liang C, Wang D, Qiu JL, Zhang F, Gao C. Cytosine, but not adenine, base editors induce genome-wide offtarget mutations in rice. Science. 2019;364(6437):292-5.

15. Zhang M, Zhou C, Wei Y, Xu C, Pan H, Ying W, et al, Human cleaving embryos enable robust homozygotic nucleotide substitutions by base editors.Data sets. SRA. 2019. https://www.ncbi.nlm.nih.gov/sra/SRP136376.

Ready to submit your research? Choose BMC and benefit from:

- fast, convenient online submission

- thorough peer review by experienced researchers in your field

- rapid publication on acceptance

- support for research data, including large and complex data types

- gold Open Access which fosters wider collaboration and increased citations

- maximum visibility for your research: over $100 \mathrm{M}$ website views per year

At $\mathrm{BMC}$, research is always in progress.

Learn more biomedcentral.com/submissions 